\title{
A novel tsRNA-16902 regulating the adipogenicdifferentiation of human bone marrow mesenchymal stem cells
}

\section{Tao Wang ( $\nabla$ comwangtaocom@163.com )}

Jiujiang University Medical College

\section{Jun Mei}

Jiujiang University

\section{Xing-nuan Li}

Jiujiang University

\section{Xiao-yuan Xu}

Jiujiang University

\section{Bai-cheng Ma}

Jiujiang University

\section{Wei-dong Li}

Jiujiang University

\section{Research}

Keywords: tRNA-derived small RNAs (tsRNAs), Human bone marrow mesenchymal stem cells (hMSCs), adipogenic differentiation, retinoic acid receptor $\mathrm{Y}$ (RARY), Smad 2/3 signaling pathway

Posted Date: July 27th, 2020

DOI: https://doi.org/10.21203/rs.3.rs-31276/v2

License: (c) (1) This work is licensed under a Creative Commons Attribution 4.0 International License. Read Full License

Version of Record: A version of this preprint was published on August 24th, 2020. See the published version at https://doi.org/10.1186/s13287-020-01882-6. 


\section{Abstract}

Background: Transfer RNA-derived small RNAs (tsRNAs) are a recently discovered form of non-coding RNA capable of regulating myriad physiological processes. The role of tsRNAs in hMSC adipogenic differentiation, however, remains incompletely understood. The purpose of this study was to identify the novel tsRNA-16902 as a regulator of hMSC adipogenic differentiation.

Methods: In this study we conducted transcriptomic sequencing of hMSCs after inducing their adipogenic differentiation, and we were thereby able to clarify the molecular mechanism underlying the role of tsRNA-16902 in this context via a series of molecular biology methods.

Results: When we knocked down tsRNA-16902 expression, this impaired hMSC adipogenic differentiation and associated marker gene expression. Bioinformatics analyses further revealed tsRNA-16902 to target retinoic acid receptor $Y$ (RARY). Luciferase reporter assays also confirmed the ability of tsRNA-16902 to bind to the RARY 3'-untranslated region. Consistent with this, RARy overexpression led to impaired hMSC adipogenesis. Further analyses additionally revealed that Smad2/3 phosphorylation as increased in cells that either overexpressed RARY or in which tsRNA-16902 had been knocked down. We also assessed the adipogenic differentiation of hMSCs in which tsRNA-16902 was knocked down and at the same time a Smad2/3 inhibitor was added to disrupt Smad2/3 phosphorylation. The adipogenic differentiation of hMSCs in which tsRNA-16902 was knocked down was further enhanced upon the addition of a Smad2/3 signaling inhibitor relative to tsRNA-16902 knockdown alone.

Conclusions: Through a comprehensive profiling analysis of tsRNAs that were differentially expressed in the context of hMSC adipogenic differentiation, we were able to identify tsRNA-16902 as a previously uncharacterized regulator of adipogenesis. tsRNA-16902 is able to regulate hMSC adipogenic differentiation by targeting RARY via the Smad2/3 signaling pathway. Together our results may thus highlight novel strategies of value for treating obesity.

\section{Background}

Adipogenesis is a mechanism whereby pre-adipocytes undergo differentiation and maturation into adipocytes capable of mediating fat storage[1] . Dysfunctional adipogenesis can drive obesity and associated metabolic disorders including type II diabetes mellitus and cardiovascular disease[2,3]. It is therefore important that the adipogenic process be better understood in an effort to help combat these serious illnesses. Human bone marrow mesenchymal stem cells (hMSCs) are self-renewing multipotent stem cells which can differentiate into many different cell types[4], including adipocytes[5], chondrocytes[6], and osteoblasts[7]. Owing to their robust proliferation and multipotency, hMSCs are frequently utilized for in vitro studies of the mechanisms of adipogenic differentiation[8].

There is increasingly strong evidence demonstrating that small non-coding RNAs (sncRNAs) can serve as key regulators of myriad biological processes including cell differentiation, proliferation[9,10], and stress responses[11]. Studies of sncRNAs including the well-characterized microRNAs (miRNAs) have shown 
that these molecules can have a strong impact on biological systems through their ability to modulate target gene expression[12]. Further work has highlighted the potential value of such sncRNAs as tools for molecular biology and for therapeutic use as a means of silencing target gene expression in a targeted manner in contexts where suppressing such gene expression may be advantageous[13].

Improved sequencing platforms and approaches have recently led researchers to understand that tRNA loci can also give rise to sncRNA molecules, with these tRNA fragments (tRFs), which are also known as tRNA-derived small RNAs (tsRNAs), serving as a recent area of significant research interest[14-17]. These tsRNAs have been grouped into 6 different classes that are defined according to their specific tRNA origins (Fig.1) [18-20]. Cleavage of tRNAs at the anticodon site by angiogenin yields 5'- and 3'-tRNA halves, whereas mature tRNA cleavage at the D-loop or anticodon step yields tRFs- 5 , and cleavage at the T-loop or anticodon stem yields tRFs-3. Fragments from the internal portions of mature tRNAs are referred to as internal tRFs (i-tRFs), while tRFs-1 are generated from 3' end fragments of primary tRNAs.

Given that tsRNA expression has been detected in myriad biological contexts, there has been substantial interest in exploring the relevance of these molecules to normal physiology and to the development of disease. Specific tsRNAs have been found to function through a range of mechanisms in order to suppress oncogenesis[21], regulate cancer cell ribosomal biogenesis[22], influence paternal epigenetic inheritance[23-24], and facilitate LTR-retrotransposon control[25]. The specific role of tsRNAs in hMSC adipogenic differentiation, however, has yet to be firmly established.

In the present study we employed a high-throughput sequencing approach in order to identify those tsRNAs that were differentially expressed during the adipogenic differentiation of hMSCs. We then analyzed these tsRNAs in a series of bioinformatics analyses aimed at unraveling their functional importance in the context of adipogenesis. Through this approach we were able to identify the novel tsRNA-16902 (a 3'-tRNA half) as a tsRNA that regulates hMSC adipogenic differentiation by targeting retinoic acid receptor $Y$ (RARY) via the Smad 2/3 signaling pathway.

\section{Materials And Methods}

\section{Cell culture and adipogenesis}

hMSCs (HUXMA-01001, Cyagen Biosciences, China; cell lot no. of three donors: 150724I31, 160202I31, and 161125R41) were phenotyped via flow cytometry and were confirmed to be $\geq 95 \%$ CD73-, CD90-, and CD105-positive, as well as CD11b-, CD19-, CD45-, CD34-, and CD HLA-DR-negative ( $\leq 5 \%)$ as shown in additional File S1 (Supplementary Files). hMSCs were cultured at $5 \times 10^{4} \mathrm{cells} / \mathrm{cm}^{2}$ in OriCell hMSC Growth Media (HUXMA-90011; Cyagen Biosciences), supplemented with L-glutamine, 10\% FBS, and penicillin/streptomycin. Cells were harvested using trypsin-EDTA (Invitrogen, CA, USA) and were passaged until reaching the sixth passage at which time they were used for experimentation.

Adipogenesis was induced by replacing normal hMSC growth media with DMEM, containing $10 \% \mathrm{FBS}$, $10 \% \mathrm{FBS}, 10 \% \mathrm{~g} / \mathrm{mL}$ insulin, $0.5 \mathrm{mM}$ 3-isobutyl-1-methylxanthine, and $0.5 \mathrm{mM}$ dexamethasone after cells 
had reached confluence[26]. These cells were then cultured in this media for $0,7,14$, or 21 days after which they underwent high-throughput sequencing analyses.

\section{Library construction and small RNA-seq}

Cellular RNA was extracted using Trizol (Invitrogen), with RNA quality confirmed using an Agilent 2200 machine. Samples that had an RNA integrity (RIN) score>7.0 were then used for cDNA library preparation with the NEBNext Small RNA Library Prep Kit for Illumina. This RNA was first ligated to provided 3' and 5' adapters, after which first strand cDNA synthesis was performed. Index sequences and Illumina sequence adapters were then applied through index PCR, after which the library was purified, assessed for quality control with a Bioanalyzer 2200 instrument (Agilent, CA, USA), and sequences using a HiSeq X-ten platform (Illumina, CA, USA) with 150 bp paired-end reads.

\section{Small RNA analysis}

Low quality reads and adapter sequences were removed from raw data using Trim Galore, after which all remaining sequences from 12 - 50 nucleotides long were subjected to sequence alignment with those sequences found in miRBase (http://www.mirbase.org/). Identification of known miRNAs was conducted using BWA after which unmapped reads were aligned to rRNA sequences (https://rnacentral.org/). Next, internally designed tRNA sequence database (using sequences from http://gtrnadb.ucsc.edu/ and https://cm.jefferson.edu/MINTbase/) was used to analyze any remaining unmapped reads, First, intronic sequences were removed after which CCA was added to the end of each tRNA sequence. A total of 50 genomic nucleotides were then added behind these CCA residues, with the resultant mapped reads being identified as potential tsRNAs that were then classified using tRFdb (http://genome.bioch.virginia.edu/trfdb/) and MINTBase (https://cm.jefferson.edu/MINTbase/).

\section{Differential small RNA expression and target gene prediction}

The EB-Seq algorithm[27] was used to identify those tsRNAs that were differentially regulated during adipogenesis based upon P-value and FDR significance analyses[28], with the cutoff criteria for differentially regulated sncRNAs being: i) Fold Change $>2$ or $<0.5$; ii) $P<0.05, F D R<0.05$. Next, putative tsRNA and miRNA target genes were identified using the miRanda[29] and RNAhybrid tools[30]. A sequencing data analysis flowchart was shown in Fig. S1 (Supplementary Files).

\section{Lentiviral transduction and hMSC screening}

Knockdown of tsRNA-16902 and overexpression of RARy was conducted using lentiviral particles from Shanghai Genechem Co., Ltd. A short hairpin RNA (shRNA) targeting tsRNA-16902 was designed (target sequence,5'-TGGTGTCCTTGGAAAAAGGTTTTCATCTCCGGTTTACAA-3'). In addition, a negative control (NC) shRNA was used (sequence, 5'-TTCTCCGAACGTGTCACGT-3'). Lentiviral transduction reactions were conducted by plating $5 \times 10^{4} \mathrm{hMSCs} / \mathrm{cm}^{2}$ in 6-well plates until $20-30 \%$ confluent, after which they were infected using $10 \mathrm{uL}$ of the appropriate lentivirus $\left(1 \times 10^{8}\right.$ infectious units $\left./ \mathrm{ml}\right)$ in complete media 
supplemented with $5 \mu \mathrm{g} / \mathrm{ml}$ polybrene. After $10 \mathrm{~h}$, this transduction media was removed and fresh media was added, after which the cells were grown for an additional $72 \mathrm{~h}$. Cells were then cultured in $0.5 \mu \mathrm{g} / \mathrm{ml}$ puromycin for $48 \mathrm{~h}$, after which they were screened over a 6 day period with fresh media added every 1-2 days.

At various time points during adipogenesis, Oil Red $O$ staining was used to confirm the presence of lipid droplets within cells. In addition, cells were collected to assess the expression of adipogenic markers and RARY.

\section{Oil red 0 staining}

Cells were washed with PBS and were then fixed with $10 \%$ formalin for 30 min, after which they were washed using $60 \%$ isopropanol prior to staining for 10 minutes with Oil red 0 (0.3\%; Sigma-Aldrich) while shaking gently. Cells were then washed using distilled water to remove free dye. In addition, free dye was eluted from these cells using $100 \%$ isopropanol, after which absorbance at $490 \mathrm{~nm}$ was assessed via spectrophotometry[31].

\section{qRT-PCR}

TRIzol (Invitrogen) was used to extract cellular RNA as above, and a cDNA Reverse Transcription Kit (Thermo, CA, USA) was then utilized for CDNA preparation. All qRT-PCR reactions were conducted using a SYBR Premix Ex Taq kit (Toyobo, Osaka, Japan) with an ABI Prism 7500 instrument (Applied Biosystems). The primers were used in this study are compiled in Table S1 (Supplementary Files). Relative gene expression was quantified using the $2^{-\Delta \Delta C t}$ method[32-33].

\section{Western blotting}

Cells were lysed using RIPA buffer, after which 15 ug of protein per sample was boiled in 5×SDS sample buffer for 5 minutes, separated via 10\% SDS-PAGE, and transferred to a PVDF membrane (Millipore). Blots were blocked with $5 \%$ non-fat milk for $2 \mathrm{~h}$, and were probed overnight with the following antibodies: rabbit anti-PPARY (abcam 191407), rabbit anti- C/EBPa (abcam 40764), rabbit anti-FABP4 (abcam 92501), rabbit anti-RARY (abcam 191368), rabbit anti-Smad2/3(abcam 63672), rabbit anti-p-Smad2/3 (abcam 63399) and mouse anti- $\beta$-actin (1:2,000; abcam 173838). All rabbit antibodies were diluted 1:1,000. Blots were then probed for $1 \mathrm{~h}$ with an appropriate secondary HRP-linked antibody (1:5,000; CST), after which enhanced chemiluminescence (BeyoECL Plus; Beyotime Institute of Biotechnology) was used for protein visualization.

\section{Luciferase reporter assay}

Briefly, a WT or mutated version of the RARY 3 '-UTR sequence containing this potential tsRNA binding site was cloned into the pGL3 vector downstream of luciferase, after which DNA sequencing was used to confirm construct identity. This vector was then co-transfected into 293T cells with or without a tsRNA- 
16902 mimic. After $48 \mathrm{~h}$, the luciferase activity in these cells was quantified with Renilla luciferase activity being used for normalization purposes.

\section{Statistical analysis}

SPSS v16.0 (SPSS, IL, USA) was used for statistical testing. Data are given as means \pm standard deviation (SD). Data were compared using Student's t-tests and one-way ANOVAs, as appropriate, with a two-tailed $P<0.05$ as the significance threshold.

\section{Results}

\section{Characterization of the tsRNA expression profile of hMSCs undergoing adipogenesis}

We began by assessing which tsRNAs were differentially expressed in hMSCs during the process of adipogenic differentiation by collecting RNA from these cells at days $0,7,14$, and 21 of adipogenesis and conducting small RNA sequencing using an Illumina HiSeq $X$ ten platform. The GtRNA and piRNA databases were then used to filter out reads from 24 - 33 nucleotides long which were then aligned with those in tRFdb and tRF MINTbase to yield sample tsRNA expression profiles. These identified tsRNAs were primarily from 17-23 or 30-36 nucleotides long at all time points (Fig. 2a). In addition, the majority of 5'-tRNA halves, tRFs-5, 3'-tRNA halves, and tRFs-3 had positions similar to those of the parental tRNAs from which they were derived (Fig. 2b).

Relative to baseline, we found that the relative frequency of these tsRNAs as a fraction of total noncoding RNAs was significantly increased at all tested time points during adipogenic differentiation (Fig. 2c). All six primary classes of tRFs were detectable in these samples, with the relative frequencies of individual tRF subtypes varying over the course of adipogenesis and with the most significant increases being evident on days 7 and 14 of this process (Fig.2d).

\section{Identification of tsRNAs differentially expressed during adipogenesis}

We next utilized the EBSeq algorithm in order to identify those tsRNAs that were differentially expressed during hMSC adipogenesis, with Log2FC $>1$ and FDR $<0.05$ as cut-off criteria in order to identify differentially expressed tsRNAs when comparing samples collected on days 0 and 7,7 and 14, or 14 and 21. We found that the peak expression of most tsRNAs occurred on day 7 of adipogenesis, and as such we therefore focused specifically on the comparison of differential tsRNA expression between day 0 and day 7 (Fig. 3a-b). In total we assessed six differentially expressed tsRNAs at this time point through preliminary experiments and found that the effect of tsRNA-16902 on adipogenic differentiation was more significant as compared to several other tested tsRNAs. Therefore we focused specifically on tsRNA-16902 (3'-tRF half; Database_ID: tRF-39-ZLBS5EOB3ZY61DE2).

\section{Knockdown of tsRNA-16902 inhibitshMSC adipogenesis}


We next generated hMSCs that were stably transduced to an shRNA specific for tsRNA-16902 or to overexpress RARy. After 6 days of puromycin selection, stably transduced cells that proliferated effectively and expressed GFP were obtained consistent with successful stable transduction (Fig. 4).

We began by exploring the functional importance of tsRNA-16902 in the context of hMSC adipogenesis by knocking down this tsRNA and assessing adipogenic differentiation of these hMSCs after 0, 7, and 14 days. Through Oil Red $O$ staining we determined that knockdown of this tsRNA significantly suppressed lipid accumulation within these cells consistent with disrupted adipogenesis (Fig. 5a). This was further confirmed when isopropanol elution was used to quantify the intensity of Oil Red 0 staining in these negative control or tsRNA-16902-knockdown cells (Fig. 5b).

We next examined the impact of tsRNA-16902 knockdown on the expression of the adipogenic markers PPARY, FABP4 and C/EBPa at the RNA and protein levels. We found that knocking down this tsRNA was associated with significant reductions in the expression of all three of these markers (Fig. $5 c-d$ ), thus suggesting that tsRNA-16902 plays a central role in adipogenesis.

\section{RARY is a directtsRNA-16902 target}

We next sought to identify potential tsRNA-16902 target genes with the miRanda and RNAhybrid databases, which identified RARy as a putative target of this tsRNA. We then sought to test the importance of RARY in the regulation of hMSC adipogenesis by overexpressing this gene in hMSCs. We found that overexpressing RARy significantly disrupted adipogenesis on days 7 and 14 (Fig. 6a-b), and that this also corresponded to significant reductions in the expression of PPARY, C/EBPa, and FABP4 (Fig. $6 c-d)$.

We next explored whether tsRNA-16902 was able to directly interact with the RARy mRNA in order to better characterize the regulatory relationship between these molecules in the context of adipogenesis. We therefore began by assessing how tsRNA-16902 influenced RARy expression during the adipogenic process (Fig. 7a). We then used Targetscan in order to identify the predicted tsRNA-16902 binding site within the RARY 3'-UTR (Fig. 7b). To confirm the validity of this binding site, a luciferase reporter assay was then conducted which revealed that tsRNA-16902 transfection into cells resulted in a $50 \%$ reduction in luciferase activity for constructs containing a WT but not a mutated version of this RARY 3'-UTR binding site (Fig. 7c).

\section{Both tsRNA-16902 knockdown and RARyoverexpressionincreaseSmad2/3 phosphorylation}

We finally explored the ability of altered RARy and tsRNA-16902 expression to impact Smad2/3 signaling in the context of adipogenesis by measuring Smad2/3 phosphorylation by Western blotting at different time points. These analyses revealed that both tsRNA-16902 knockdown and RARy overexpression were associated with a significant increase in p-Smad2/3levels (Fig. 8a-b).

\section{A Smad2/3 inhibitor reverses knockdown tsRNA-16902-mediated hMSC adipogenic differentiation}


We additionally treated cells with a Smad2/3 inhibitor (TP0427736, Selleck) in order to reduce pSmad2/3 levels in these cells (Fig. 8c), revealing that adipogenic differentiation in hMSCs in which tsRNA-16902 had been knocked down was more robust as compared with that in hMSCs in which tsRNA16902 had been knocked down in the absence of inhibitor addition (Fig. 9). These results thus further revealed that the Smad2/3 signaling pathway is downstream of tsRNA-16902.

\section{Discussion}

The ability of diverse non-coding RNA molecules to regulate adipogenesis has been increasingly well documented in recent years[34-36]. In addition better-studied molecules such as miRNAs, IncRNAs and circRNAs, many other non-coding RNA subtypes, including tsRNAs, have also been identified and shown to be biologically important in diverse contexts[21-25]. The role of tsRNAs in hMSC adipogenic differentiation, however, remains incompletely understood.

In this study we began by characterizing tsRNA expression profiles during hMSC adipogenic differentiation. This analysis revealed that tsRNAs expressed in these cells were primarily from 17-23 and 30-36 nucleotides in length, thus strongly suggesting that these tRNA segments were not the products of random degradation (Fig. 2a). In addition, the majority of 5'-tRNA halves, tRFs-5, 3'-tRNA halves, and tRFs3 had positions similar to those of the parental tRNAs from which they were derived (Fig. 2b), further confirming that these molecules resulted from specific cleavage events. These findings were thus consistent with previous studies of tsRNA biology[21-22,37].

All 6 tsRNA subtypes were evident within our samples, with the majority of these tsRNAs reaching peak expression levels at day 7 of the adipogenic process before declining on days 14 and 21 (Fig. 2c-d). This suggested a potentially pivotal role for these tsRNAs in adipogenesis. We therefore focused on differential tsRNA expression on day 7, as this was both the time point at which most tsRNAs were maximally expressed and a time point known to be critical for pre-adipocyte fate determination in this context[38-39].

We next assessed patterns of differential tsRNA expression at this day 7 time point (Fig. 3a-b), revealing 6 tsRNAs that were not detectable on day 0 and yet reached peak expression on day 7 . This suggested that these tsRNAs may thus be key regulators of adipogenesis, and as such we next focused on one of these tsRNAs in follow-up experiments. We found that knocking down tsRNA-16902 inhibited hMSC adipogenesis, implying that tsRNA-16902 may promote adipogenic differentiation in this context (Fig. 5). Given the clear impact of tsRNA-16902 knockdown on hMSC adipogenesis, we then studied this tsRNA further.

tsRNAs have been shown to play evolutionarily-conserved roles in stress response in eukaryotes. [40-42]. tsRNAs have also been shown to play an essential role in the context of cancers, neurological disease, and metabolic disorders[21-25]. In one recent report, researchers found that tRF ${ }^{\text {GluTTC }}$ was capable of inhibiting 3T3-L1 preadipocyte differentiation into adipocytes[43]. Interestingly, we identified a distinct 
tsRNA that was able to modulate hMSC adipogenesis, suggesting that these tsRNAs may function via distinct mechanisms in this context. We also utilized primary hMSCs rather than the 3T3-L1 cell line model, potentially indicating that our results are more physiologically relevant. Other studies have highlighted the ability of tsRNAs to regulate stem cell states in murine embryonic stem cells (mESCs), consistent with our findings[44]. As tsRNAs are a relatively recent area of research, there have been relatively few studies of them to date in vivo[25,43-44]. As we utilized human bone marrow mesenchymal stem cells as research subjects in our study, further in-vivo validation of our findings will be challenging. However, studies of the adipogenic differentiation of human bone marrow mesenchymal stem cells are common as these cells represent an ideal, and as such our results are still of great reference value and significance[5,6,8]. Although no further in-vivo validation experiments were performed in our study, we believe that our data clearly show that tsRNA is likely to play an important role in the adipogenic differentiation process.

How tsRNA-16902 controls hMSC adipogenic differentiation remains to be fully clarified. Several studies to date have proposed distinct mechanisms whereby tsRNAs control gene expression[45-47]. One study suggests that certain tsRNAs may function in a manner similar to miRNAs, guiding Argonaute(Ago) to regulate target gene expression[48]. However, these studies provide contradictory evidence suggesting that tsRNAs and miRNAs function via distinct mechanisms, with tsRNA target sites being present not just in the 3'-UTR but also in the 5'-UTR and CDS regions of target mRNAs[49]. In this study we identified putative tsRNA-16902 target genes based upon their sequence complementarity, thus identifying RARy as a putative target of this tsRNA (Fig. 7a).

Retinoic acid (RA) is a key regulator of cell differentiation in many contexts[50-52], functioning so as to control gene expression via activation of the RA receptor (RAR) and retinoid X receptor (RXR) families of nuclear hormone receptors[53]. RARy activation has been shown to be important for inhibiting PPARY2 expression in 3T3-L1 adipocyte differentiation[54]. This thus suggests that RARy may be an important regulator of adipogenesis. Consistent with this, we found that RARY overexpression impaired hMSC adipogenesis (Fig. 6), thus confirming its relevance in this process. We further found that the expression of tsRNA-16902 and RARy were negatively correlated with one another (Fig. 7b). Using a luciferase reporter assay we then further confirmed that RARy was a direct target of tsRNA-16902 (Fig. 7c).

It is well known that there are many signaling pathways that affect the adipogenic differentiation of hMSCs. Selecting the appropriate signaling pathway downstream of RARY is very important. The Smad2/3 signaling pathway has recently been shown to be central to the regulation of adipogenesis[5557]. In addition, this signaling pathway involved in non-coding RNA has also been shown to play an essential role in the context of cancers, cell differentiation, and migration[58-60]. Interestingly, the relevant signaling pathways whereby tsRNAs modulate cellular functionality have only been analyzed via bioinformatics approaches[61,62], suggesting that much research remains to be conducted in this area. Therefore, the relationship between tsRNA-16902 and Smad2/3 signaling pathway was a topic of interest in the present study. We found that both tsRNA-16902 knockdown and RARy overexpression resulted in enhanced Smad2/3 phosphorylation in hMSCs (Fig. 8a, b). Furthermore, by adding a Smad2/3 inhibitor 
to suppress p-Smad2/3 levels (Fig. 8c), we were able to demonstrate that the adipogenic differentiation of hMSCs in which tsRNA-16902 was also knocked down was more robust than that in cells in which tsRNA-16902 was knocked down but to no inhibitor was added (Fig. 9). These results thus further confirmed that the Smad2/3 signaling pathway is downstream of RARy, with tsRNA-16902 controlling the activation of this pathway. Based on these findings, we therefore propose a model wherein tsRNA-16902 can regulate hMSC adipogenesis by targeting RARy via the Smad2/3 signaling (Fig. 10).

\section{Conclusions}

In summary, using a high-throughput sequencing analysis we assessed patterns of tsRNA expression during hMSC adipogenesis. This approach led us to identify tsRNA-16902 as a novel and important regulator of this process. These results may thus offer new opportunities for the treatment of obesity.

\section{Declarations}

\section{Acknowledgements}

The authors wish to thank Drs. Weidong Chen and Bo Zhang (Novel Bioinformatics Ltd., Co, Shanghai, China) for their technical assistance in bioinformatics analysis.

\section{Individual person's data}

Not applicable.

\section{Authors' contributions}

TW and BM conceived this study. JM, XL, XX and WL performed the experiments, collected data, and performed the data analysis. XX and BM helped perform the experiments. TW wrote the manuscript. All authors read and approved the final manuscript.

\section{Funding}

This study was supported by the National Nature Science Foundation of China (no. 81860260; 81960411).

\section{Availability of data and materials}

All data generated and/or analyzed during this study are included in this published article.

\section{Ethics approval and consent to participate}

Not applicable.

\section{Consent for publication}


Not applicable.

\section{Competing interests}

The authors declare that they have no competing interests

\section{Author details}

${ }^{1}$ Key Laboratory of System Bio-medicine of Jiangxi Province, Jiujiang University, Jiujiang 332000, China.

\section{Abbreviations}

tsRNAs: tRNA-derived small RNAs; hMSCs: Human bone marrow mesenchymal stem cells; RARY: retinoic acid receptor $\gamma$; PPARY: peroxisome proliferator-activated receptor $\gamma ; \mathrm{C} / \mathrm{EBPa}$ : CCAAT enhancer binding protein a; FABP4: Fatty Acid Binding Protein-4; qRT-PCR: Quantitative real-time polymerase chain reaction; UTR: Untranslated region.

\section{References}

1. Li H, Chen X, Guan L, Qi Q, Shu G, Jiang Q, Yuan L, Xi QY, Zhang YL. MiRNA-181a regulates adipogenesis by targeting tumor necrosis factor-alpha (TNF-alpha) in the porcine model. PLoS ONE, 2013; 8(10):e71568.

2. Kopelman PG. Obesity as a medical problem. Nature.2000;404(6778):635-43.

3. Qiu J, Zhou XG, Zhou XY, Zhu C, Shi CM, Ji CB, Cheng R, Li Y, Guo XR. Characterization of microRNA expression profiles in 3T3-L1 adipocytes overexpressing C10orf116. Mol Biol Rep. 2013; 40(11):6469-76

4. Pittenger MF, Mackay AM, Beck SC, Jaiswal PK, Douglas R, Mosca JD, Moorman MA, Simonetti DW, Craig S, Marshak DR. Multilineage potential of adult human mesenchymal stem cells. Science, 1999; 284(5411):143-7

5. Helder MN, Knippenberg M, Klein-Nulend J, Wuisman PI. Stem cells from adipose tissue allow challenging new concepts for regenerative medicine. Tissue Eng. 2007; 13(8):1799-808

6. Barry F, Boynton RE, Liu B, Murphy JM. Chondrogenic differentiation of mesenchymal stem cells from bone marrow: differentiation-dependent gene expression of matrix components. Exp Cell Res.2001;268(2):189-200

7. Arinzeh TL. Mesenchymal stem cells for bone repair: preclinical studies and potential orthopedic applications. Foot Ankle Clin. 2005;10(4):651-65

8. Subash-Babu P, Alshatwi AA. Aloe-emodin inhibits adipocyte differentiation and maturation during in vitro human mesenchymal stem cell adipogenesis. J Biochem Mol Toxicol. 2012;26(8):291-300.

9. Lee YS, Dutta A. MicroRNAs in cancer. Annu Rev Pathol. 2009; 4:199-227. 
10. Li X, Yang Y, Yan R, Xu X, Gao L, Mei J, Liu JY, Wang XP,Zhang J,Wu P, Li WD, et al. miR-377-3p regulates adipogenic differentiation of human bone marrow mesenchymal stem cells by regulating LIFR. Mol Cell Biochem. 2018; 449(1-2):295-303

11. Cai P, Piao X, Hao L, Liu S, Hou N, Wang H, Chen Q. A deep analysis of the small non-coding RNA population in Schistosoma japonicum eggs. PLoS One. 2013; 8(5): e64003

12. Bernstein E, Caudy AA. Hammond SM, Hannon GJ. Role for a bidentate ribonuclease in the initiation step of RNA interference. Nature.2001;409(6818): 363-6

13. Galasso M, Sana ME, Volinia S. Non-coding RNAs: a key to future personalized molecular therapy? Genome Med. 2010; 2(2): 12

14. Gebetsberger J, Polacek N. Slicing tRNAs to boost functional ncRNA diversity RNA Bio.2013;10(12):1798-806

15. Keam SP, Hutvagner G. tRNA-Derived Fragments (tRFs): Emerging New Roles for an Ancient RNA in the Regulation of Gene Expression. Life (Basel).2015;5(4):1638-51

16. Rashad S, Niizuma K, Tominaga T. tRNA cleavage: a new insight. Neural Regen Res.2020;15(1):4752

17. Raina M, Ibba M. tRNAs as regulators of biological processes. Front Genet. 2014;5,171.

18. Honda S, Loher P, Shigematsu M, Palazzo JP, Suzuki R, Imoto I, Rigoutsos I, Kirino Y. Sex hormonedependent tRNA halves enhance cell proliferation in breast and prostate cancers. Proc Natl Acad Sci U S A. 2015; 112, E3816-25.

19. Ivanov P, Emara MM, Villen J, Gygi SP, Anderson, P. Angiogenin-induced tRNA fragments inhibit translation initiation. Mol cell. 2011; 43(4):613-23.

20. Lee YS, Shibata Y, Malhotra A, Dutta, A. A novel class of small RNAs: tRNA-derived RNA fragments (tRFs). Genes Dev.2009; 23(22):2639-49.

21. Goodarzi H, Liu X, Nguyen HC, Zhang S, Fish L, Tavazoie SF. Endogenous tRNA-derived fragments suppress breast cancer progression via YBX1 displacement. Cell. 2015; 161(4):790 -802

22. Kim HK, Fuchs G, Wang S, Wei W, Zhang Y, Park H, Roy-Chaudhuri B, Li P, Xu J, Chu K, et al. A transfer-RNA-derived small RNA regulates ribosome biogenesis. Nature. 2017;552(7683):57-62.

23. Chen Q, Yan M, Cao Z, Li X, Zhang Y, Shi J, Feng GH, PengHY, Xudong Zhang XD, ZhangY, et al. Sperm tsRNAs contribute to intergenerational inheritance of an acquired metabolic disorder. Science. 2016;351(6271):397-400.

24. Sharma U, Conine CC, Shea JM, Boskovic A, Derr AG, Bing XY, Belleannee C, Kucukural A, Serra RW, Sun $F$, et al. Biogenesis and function of tRNA fragments during sperm maturation and fertilization in mammals. Science. 2016;351, 391-96.

25. Schorn AJ, Gutbrod MJ, LeBlanc C, Martienssen R. LTR-retrotransposon control by tRNA-derived small RNAs. Cell, 2017;170(1): 61-71.

26. Menssen A, Häupl T, Sittinger M, Delorme B, Charbord P, Ringe J. Differential gene expression profiling of human bone marrow derived mesenchymal stem cells during adipogenic development. 
BMC Genomics, 12, 461.

27. Leng N, Dawson JA, Thomson JA, Ruotti V, Rissman Al, Smits BM, Haag JD, Gould MN, Stewart RM, Kendziorski C. EBSeq: an empirical Bayes hierarchical model for inference in RNA-seq experiments. Bioinformatics, 2013; 29(8):1035-43.

28. Benjamini Y, Drai D, Elmer G, Kafkafi N, Golani, I. Controlling the false discovery rate in behavior genetics research. Behav Brain Res. 2001;125(1-2):279-84.

29. Enright AJ, John B, Gaul U, Tuschl T, Sander C, Marks DS. MicroRNA targets in Drosophila. Genome biol.2004;5(1):1-23.

30. Rehmsmeier M, Steffen P, Hochsmann M, Giegerich R. Fast and effective prediction of microRNA/target duplexes .RNA, 2004;10(10):1507-17.

31. Martella E, Bellotti C, Dozza B, Perrone S, Donati D, Lucarelli E.Secreted adiponectin as a marker to evaluate in vitro the adipogenic differentiation of human mesenchymal stromal cells.Cytotherapy.2014;16(11):1476-85

32. Kim JM, Hwang SH, Song EJ, Lee SY, Kim YD, Lee CH, Lee MK, Chang CL, Lee EY. Comparative Quantification of Plasma hnRNP B1 mRNA in Non-Small Cell Lung Cancer Patients by Real-Time PCR. Korean J Lab Med. 2009;29(3):249-55.

33. Marco ML, Kleerebezem M. Assessment of real-time RT-PCR for quantification of Lactobacillus plantarum gene expression during stationary phase and nutrient starvation. J Appl Microbiol. 2008;104(2):587-94.

34. Wei S, Du M, Jiang Z, Hausman GJ, Zhang L, Dodson MV. Long noncoding RNAs in regulating adipogenesis: New RNAs shed lights on obesity. Cell Mol Life Sci. 2016;73(10):2079-87.

35. Shen L, Gan M, Li Q, Wang J, Li X, Zhang S, Zhu L. MicroRNA-200b regulates preadipocyte proliferation and differentiation by targeting KLF4 Biomed Pharmacother. 2018; 103:1538-44.

36. Zhang Y, Yu B, Yu J, Zheng P, Huang Z, Luo Y, Luo J, Mao X, Yan H, He J, et al. Butyrate promotes slow-twitch myofiber formation and mitochondrial biogenesis in finishing pigs via inducing specific microRNAs and PGC-1 a expression. J Anim Sci. 2019;97(8):3180-92.

37. Shigematsu M, Honda S, KirinoY. Transfer RNA as asource of small functional RNA. J Mol Biol Mol Imaging. 2014;1,8

38. Park YH, Yun JI, Han NRPark HJAhn JY, Kim C, Choi JH, Lee E, Lim JM, Lee ST. Massproduction of early-stage bone-marrow-derived mesenchymal stem cells of rat using gelatin-coated matrix. Biomed Res Int. 2013; 2013,347618.

39. Martino NA, Reshkin SJ, Ciani E, Dell'Aquila ME. Calcium-sensing receptor-mediated osteogenic andearly-stage neurogenic differentiation in umbilical cord matrix mesenchymal stem cells from a large animal model. PLoS One. 2014;9: e111533.

40. Emara MM, Ivanov P, Hickman T, Dawra N, Tisdale S, Kedersha N, Hu GF, Anderson P. Angiogenininduced tRNA-derived stress-induced RNAs promote stress-induced stress granule assembly. J Biol Chem. 2010; 285(14): 10959-68. 
41. Thompson DM, Parker R.Stressing out over tRNA cleavage Cell.2009;138(2):215-9.

42. Dou S, Wang Y, Lu J. Metazoan tsRNAs: Biogenesis, Evolution and Regulatory Functions. Noncoding RNA. 2019; 5: pii: E18.

43. Shen, L, Tan, Z, Gan, M, Li, Q, Chen, L, Niu, L, et al. tRNA-Derived Small Non-

Coding RNAs as Novel Epigenetic Molecules Regulating Adipogenesis Biomolecules, 9: pii: E274.

44. Krishna S, Yim DG, Lakshmanan V, Tirumalai VKoh JL, Park JE, Cheong JK, Low JL, Lim MJ, Sze SK, et al. Dynamic expression of tRNA-derived small RNAs define cellular states EMBO Report,2019;20(7): e47789.

45. Haussecker D, Huang Y, Lau A, Parameswaran P, Fire AZ, Kay, MA. Human tRNA-derived small RNAs in the global regulation of RNA silencing.RNA.2010;16(4):673-95

46. Wang Q, Li T, Xu K, Zhang W, Wang X, Quan, J, Jin WB, Zhang MX, Fan GJ, Ming-Bo Wang MB. et al. The tRNA-Derived Small RNAs Regulate Gene Expression through Triggering Sequence-Specific Degradation of Target Transcripts in the Oomycete Pathogen Phytophthora sojae Front Plant Sci.2016;7:1938.

47. Martinez G, Choudury SG, Slotkin, RK. tRNA-derived small RNAs target transposable element transcripts.Nucleic Acids Res. 2017;45(9):5142-52 .

48. Luo S, He F, Luo J, Dou S, Wang Y, Guo A, Lu J. Drosophila tsRNAs preferentially suppress general translation machinery via antisense pairing and participate in cellular starvation response .Nucleic Acids Res.2018;46(10):5250-68.

49. Shi J, Zhang Y, Zhou T, Chen Q. tsRNAs: The Swiss Army Knife for Translational Regulation. Trends Biochem Sci. 2019; 44(3):185-9.

50. Mezquita B, Mezquita C.Two Opposing Faces of Retinoic Acid: Induction of Stemness or Induction of Differentiation Depending on Cell-Type Biomolecules,2019; 9(10):567.

51. Kanungo J. Retinoic Acid Signaling in P19 Stem Cell Differentiation. Anticancer Agents Med Chem. 2017;17(9):1184-98.

52. Sandhu HS, Bhanwer AJ, Puri S. Retinoic acid exacerbates chlorpyrifos action in ensuing adipogenic differentiation of C3H10T11/2 cells in a GSK3 $\beta$ dependent pathway. PLoS One, 2017;12(3):e0173031.

53. Leid $\mathrm{M}$, Kastner $\mathrm{P}$, Chambon P. Multiplicity generates diversity in the retinoic acid signalling pathways. Trends Biochem Sci. 1992; 17(10):427-33.

54. Wang X, Yang P, Liu J, Wu H, Yu W, Zhang T, FuH, Liu Y, Hai cx. RARY-C-Fos-PPARY2 signaling rather than ROS generation is critical for all-trans retinoic acid-inhibited adipocyte differentiation. Biochimie, 2014;106,121-30.

55. Luo H, Guo Y, Liu Y, Wang Y, Zheng R, Ban Y, Peng L, Yuan Q, Liu W. Growth differentiation factor 11 inhibits adipogenic differentiation by activating TGF-beta/Smad signalling pathway. Cell Prolif.2019;52(4):e12631.

56. Fan J, Sun Z.The Antiaging Gene Klotho Regulates Proliferation and Differentiation of AdiposeDerived Stem Cells. Stem Cells,2016;34(6):1615-25. 
57. Song NJ, Yoon HJ, Kim KH, Jung SR, Jang WS, Seo CR, Lee YM, Kweon DH, Hong JW, Lee JS. et al. Butein is a novel anti-adipogenic compound. J Lipid Res. 2013;54(5):1385-96.

58. Xu H, Sun Y, You B, Huang CP, Ye D, Chang C. Androgen receptor reverses the oncometabolite R-2hydroxyglutarate-induced prostate cancer cell invasion via suppressing the circRNA-51217/miRNA646/TGF $31 / p-S m a d 2 / 3$ signaling. Cancer Lett. 2020; 472:151-164.

59. Chen YF, Li YJ, Chou CH, Chiew MY, Huang HD, Ho JH, Chien S, Lee OK. Control of matrix stiffness promotes endodermal lineage specification by regulating SMAD2/3 via IncRNA LINC00458. Sci Adv. 2020;6(6):eaay0264.

60. Hübner M, Hinske CL, Effinger D, Wu T, Thon N, Kreth FW, Kreth S. Intronic miR-744 Inhibits Glioblastoma Migration by Functionally Antagonizing Its Host Gene MAP2K4. Cancers (Basel). 2018;10(11):400.

61. Wang X, Zhang Y, Ghareeb WM, Lin S, Lu X, Huang Y, Huang S, Xu Z, Chi P. A Comprehensive Repertoire of Transfer RNA-Derived Fragments and Their Regulatory Networks in Colorectal Cancer. J Comput Biol. 2020 May 11. doi: 10.1089/cmb.2019.0305. Online ahead of print.

62. Telonis AG, Rigoutsos I. Race Disparities in the Contribution of miRNA Isoforms and tRNA-Derived Fragments to Triple-Negative Breast Cancer. Cancer Res. 2018;78(5):1140-1154.

\section{Figures}




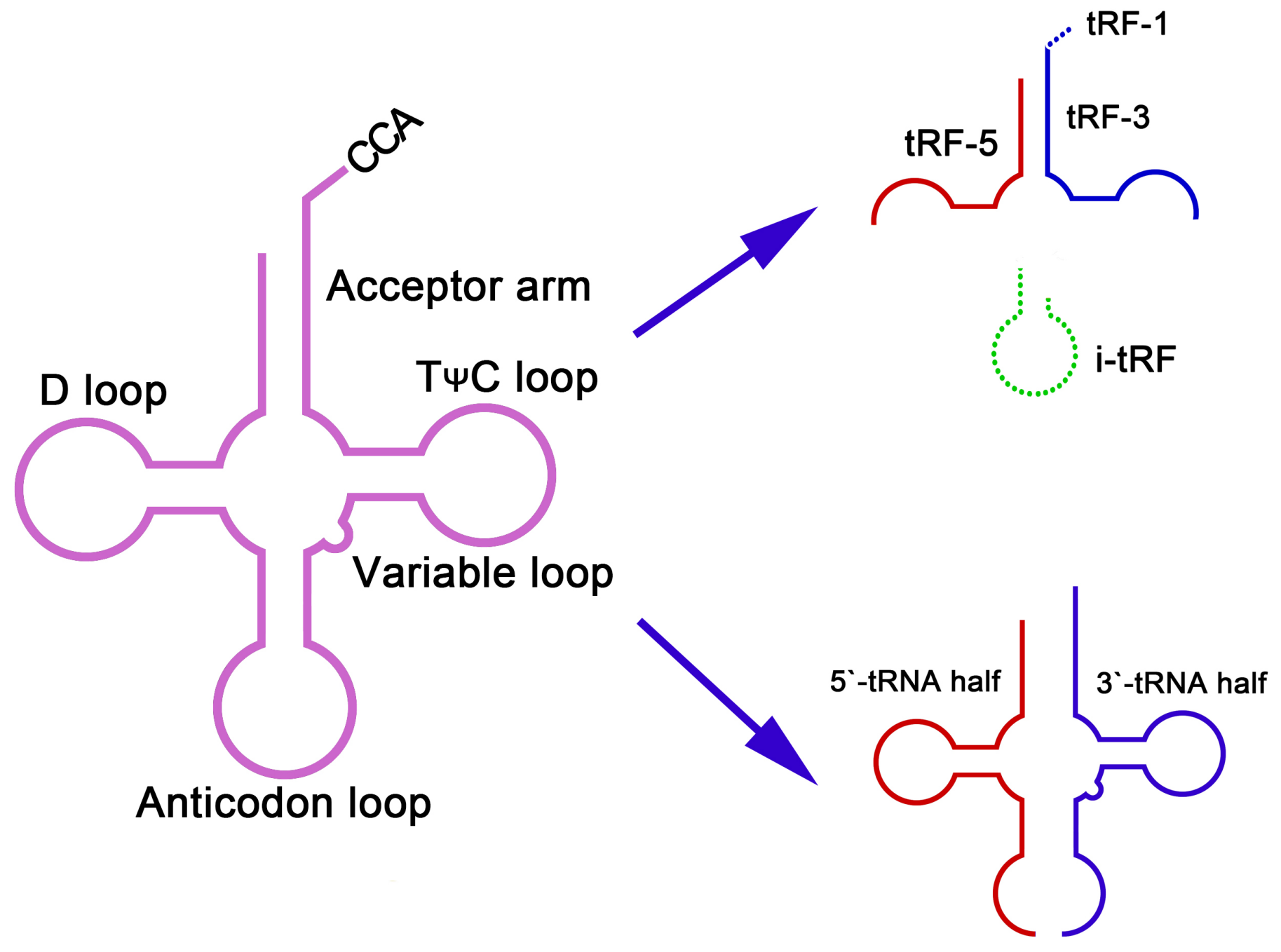

Figure 1

Different types of tRFs are produced from either the pre-tRNA or the mature tRNA. Depending on their origin and composition, these tsRNAs have been grouped into 6 different classes: tRF- 5 and tRF-3, i-tRFs, tRF-1, and 5'- and 3'-tRNA halves. 
a

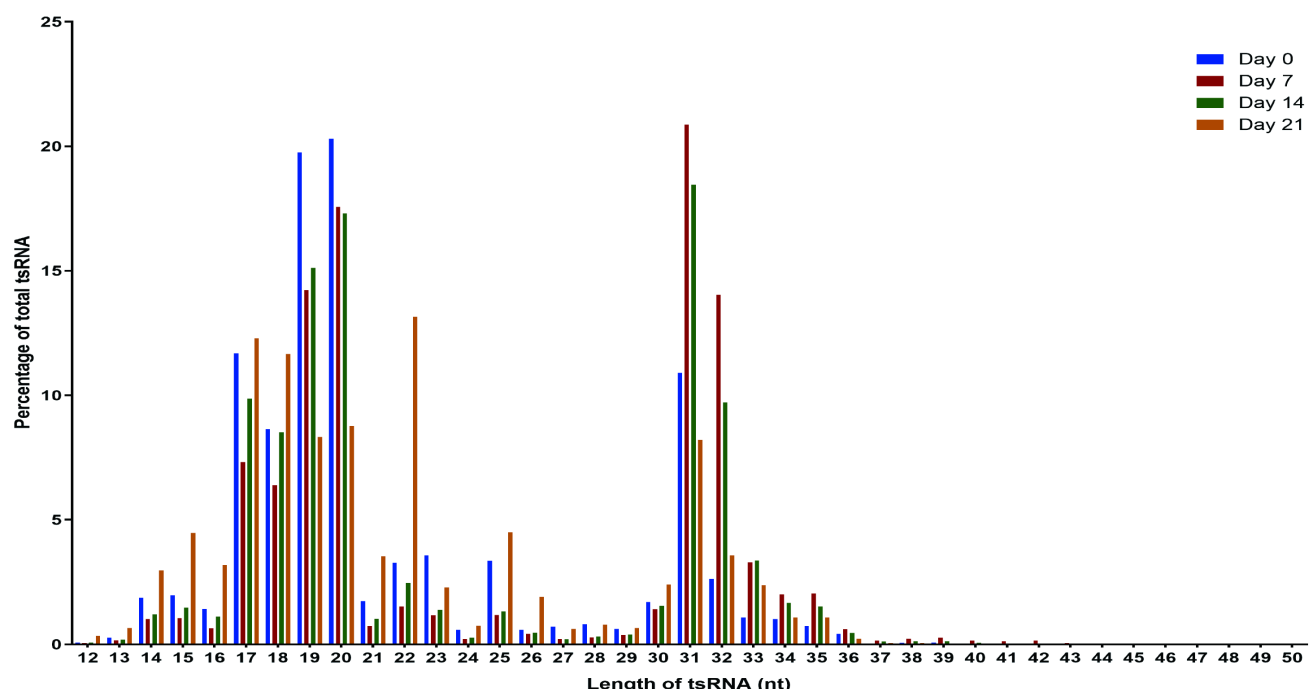

b
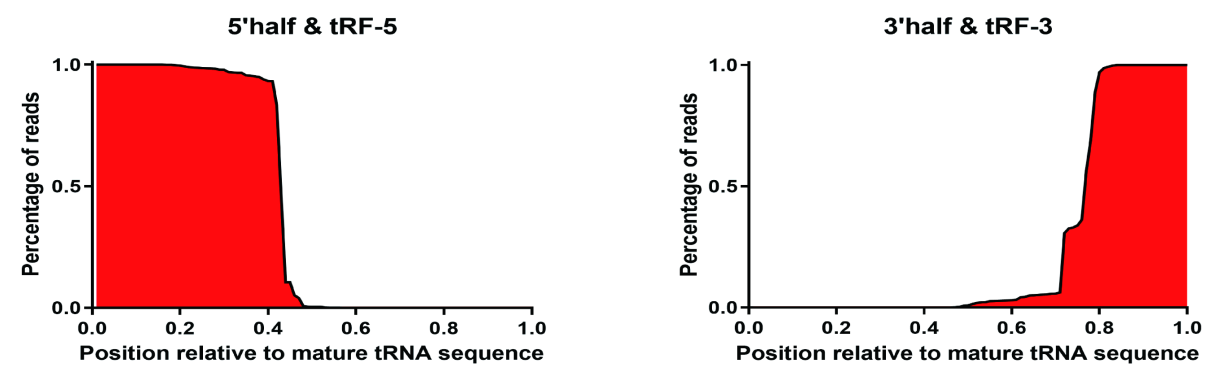

C

Day 0

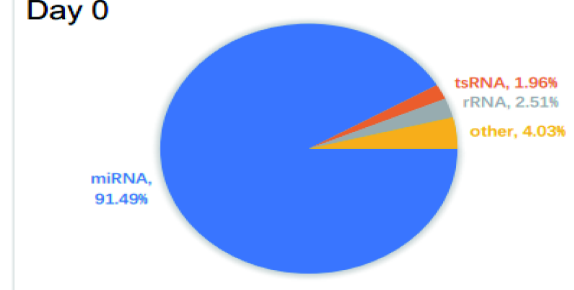

Day 14

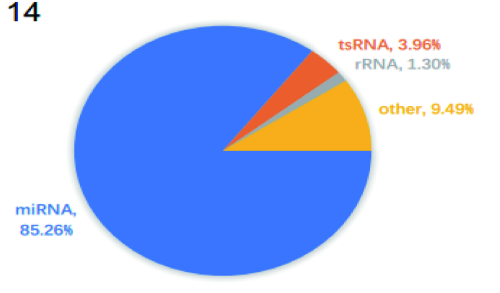

Day 7

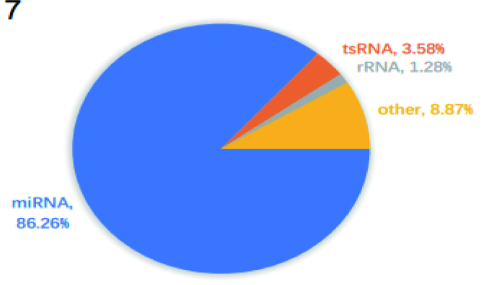

Day 21

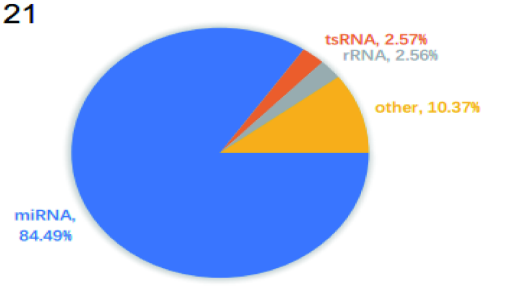

d

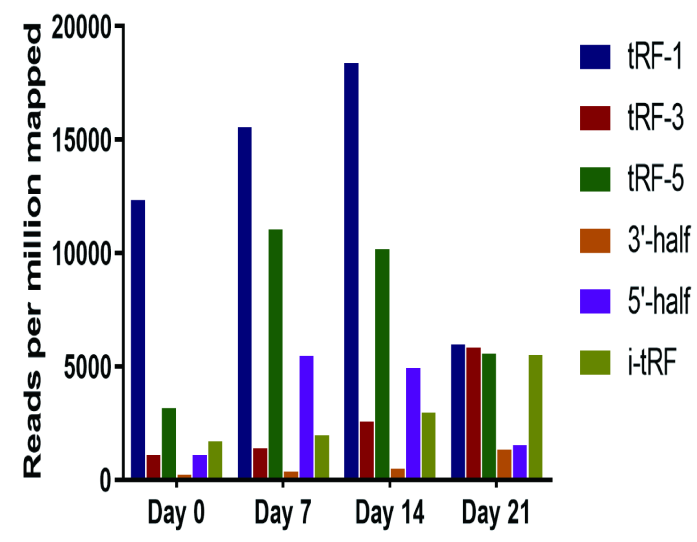

\section{Figure 2}

The expression of tsRNAs during hMSC adipogenesis. a tsRNA read distributions during adipogenic differentiation, with tsRNA length variant frequencies given as a percentage of total reads. These identified tsRNAs were primarily 17-23 or 30-36 nucleotides long at all time points. b 5'-tRF halves and tRF- 5 sequences had positions similar to those of parental tRNAs, as was true for the majority of 3 '-tRF halves and tRF-3 sequences. $c$ The frequencies of tsRNAs during adipogenesis in the overall small RNAseq, with tsRNA reads provided as a percentage of total reads. The relative frequency of these tsRNAs as a fraction of total non-coding RNAs was significantly increased at all tested time points during 
adipogenic differentiation. $d$ All six primary classes of tRFs were detectable in these samples, with the relative frequencies of individual tRF subtypes varying over the course of adipogenesis and with the most significant increases being evident on days 7 and 14 of this process.

a

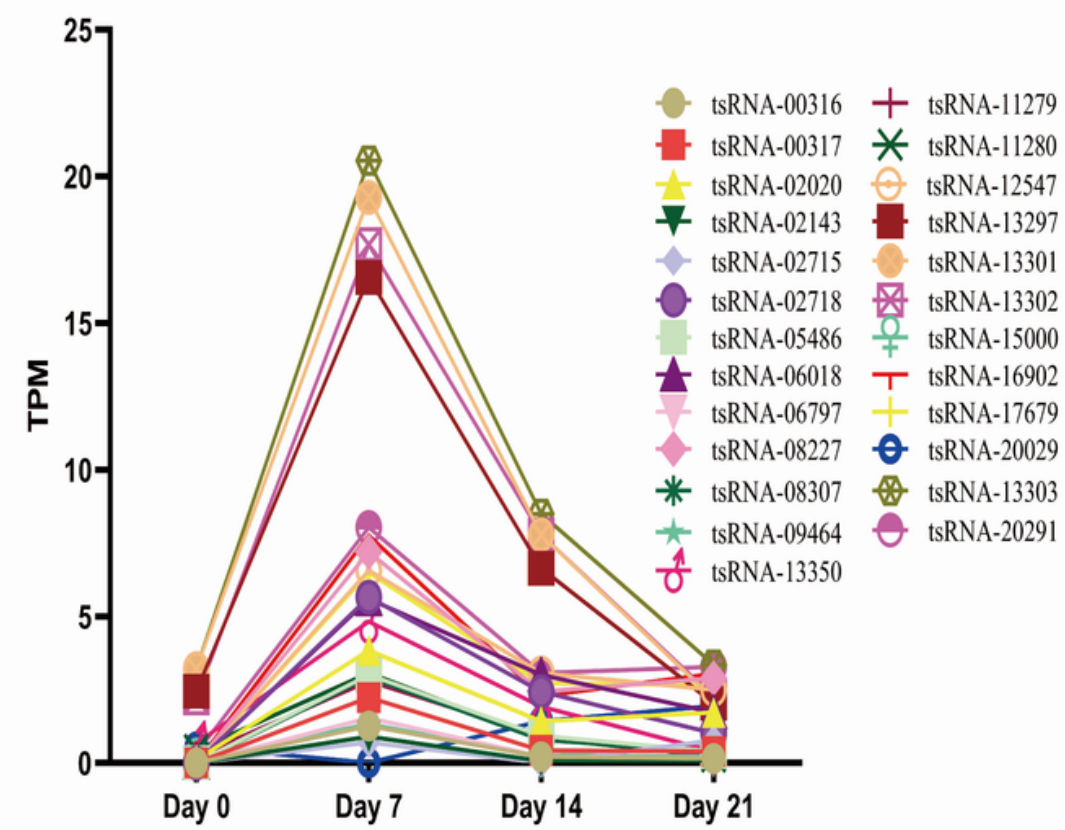

$\mathrm{b}$

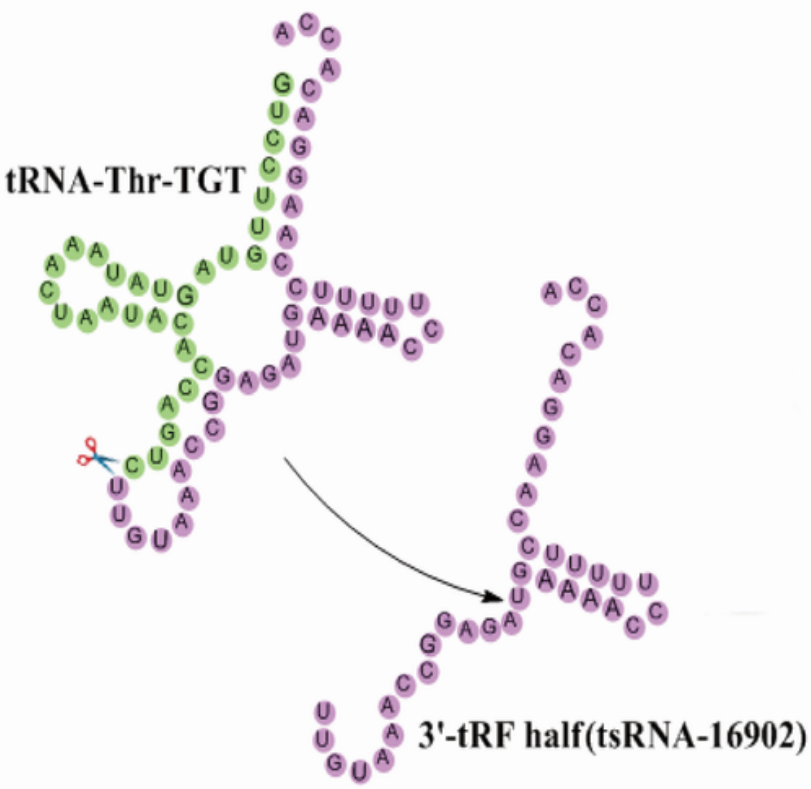

Day 0

Day 7

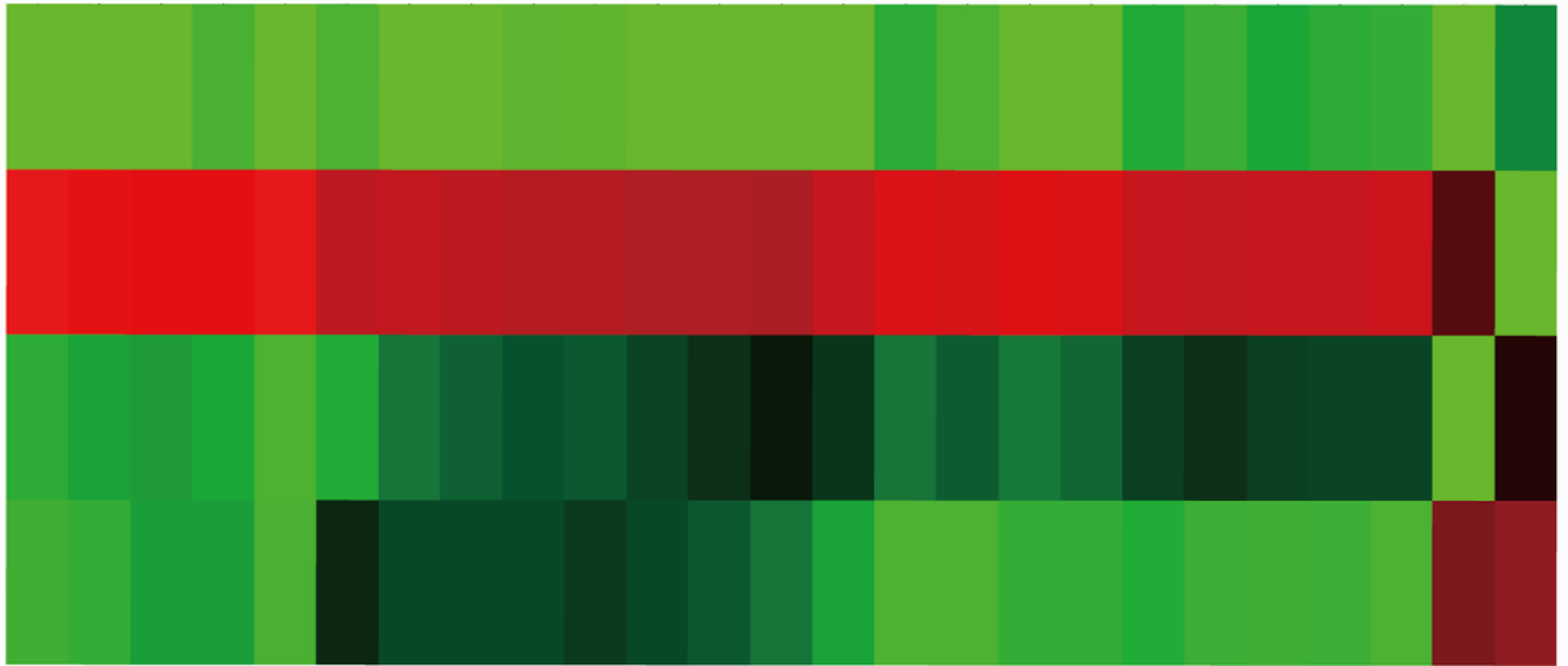

\begin{tabular}{|c|c|c|c|c|c|c|c|c|c|c|c|c|c|c|c|c|c|c|c|c|c|c|c|c|c|}
\hline 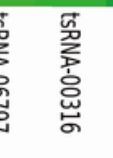 & $\begin{array}{l}\text { 苛 } \\
\text { 咅 } \\
\text { 嵌 }\end{array}$ & $\begin{array}{l}\text { 劳 } \\
\text { 辛 } \\
\text { 号 } \\
\text { 总 }\end{array}$ & 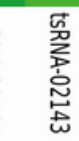 & $\begin{array}{l}\text { 量 } \\
\text { 紊 } \\
\text { 岁 } \\
\text { o }\end{array}$ & 营 & 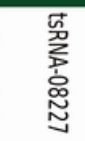 & 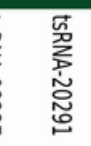 & 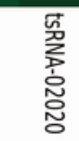 & 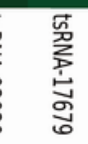 & 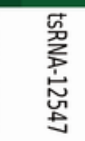 & 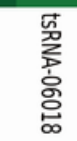 & 売 & 营 & $\begin{array}{l}\text { 袋 } \\
\text { 点 } \\
\text { 范 }\end{array}$ & 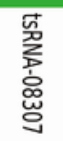 & 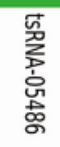 & 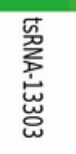 & \multicolumn{5}{|c|}{$\begin{array}{l}\text { 营 } \\
\text { 总 } \\
\text { 岕 } \\
\text { 岁 }\end{array}$} & \multicolumn{2}{|c|}{ 苞 } & 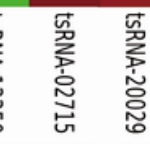 \\
\hline
\end{tabular}

\section{Figure 3}

a tsRNAs presented differential expression in the context of hMSC adipogenic differentiation. The peak expression of most tsRNAs occurred on day 7 of adipogenesis, and as such we therefore focused specifically on the comparison of differential tsRNA expression between day 0 and day 7. TPM: 
Transcripts Per Million Reads. b In total we assessed six differentially expressed tsRNAs on day 7, and focused specifically on tsRNA-16902. tRNA-Thr-TGT underwent digestion to yield a corresponding 3'-tRNA half (tsRNA-16902; Database_ID: tRF-39-ZLBS5EOB3ZY61DE2).

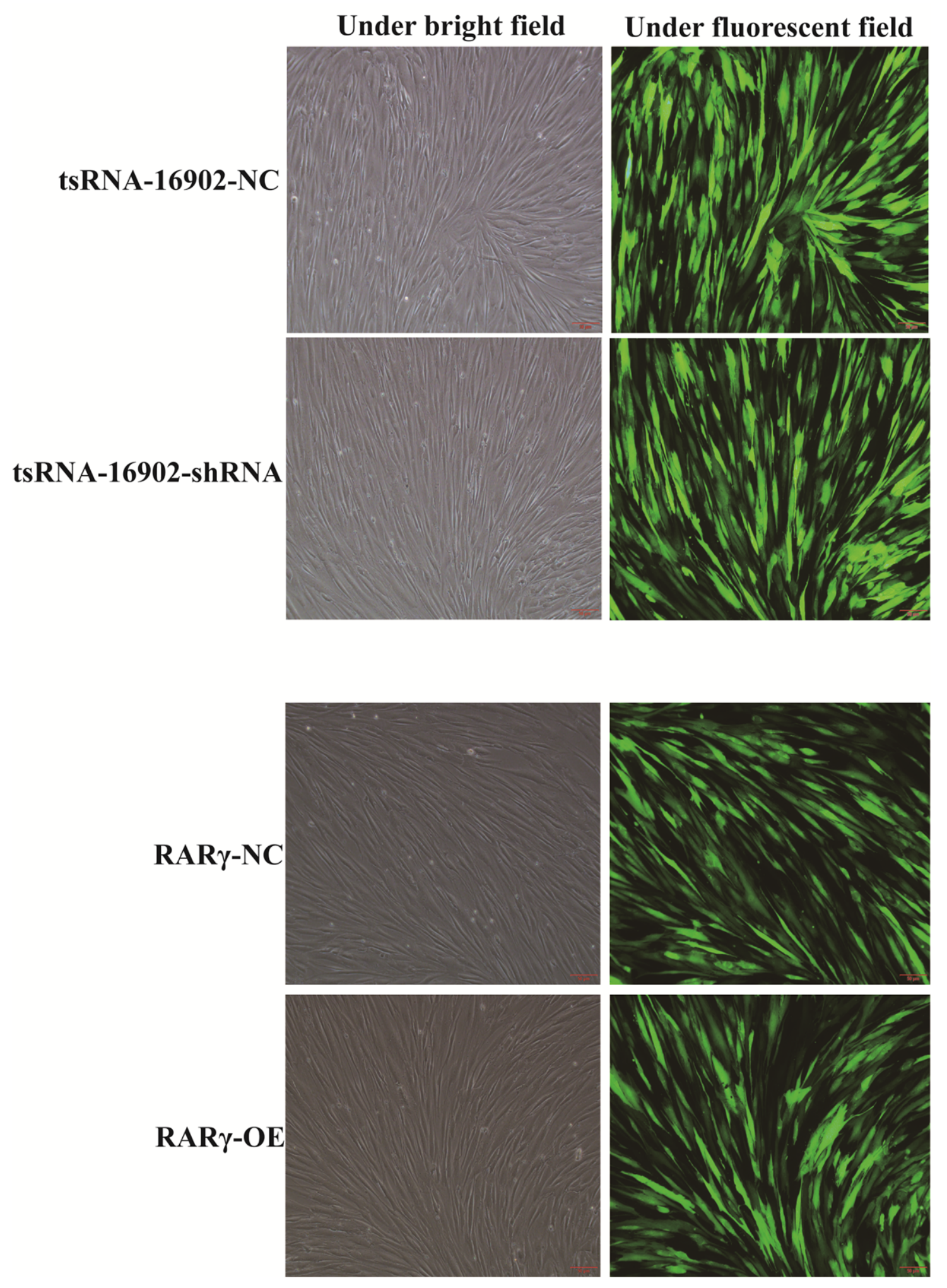

Figure 4

After 6 days of puromycin selection, stably transduced cells that proliferated effectively and expressed GFP were obtained consistent with successful stable transduction. Transduced cells were assessed via 


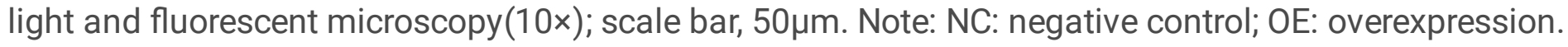
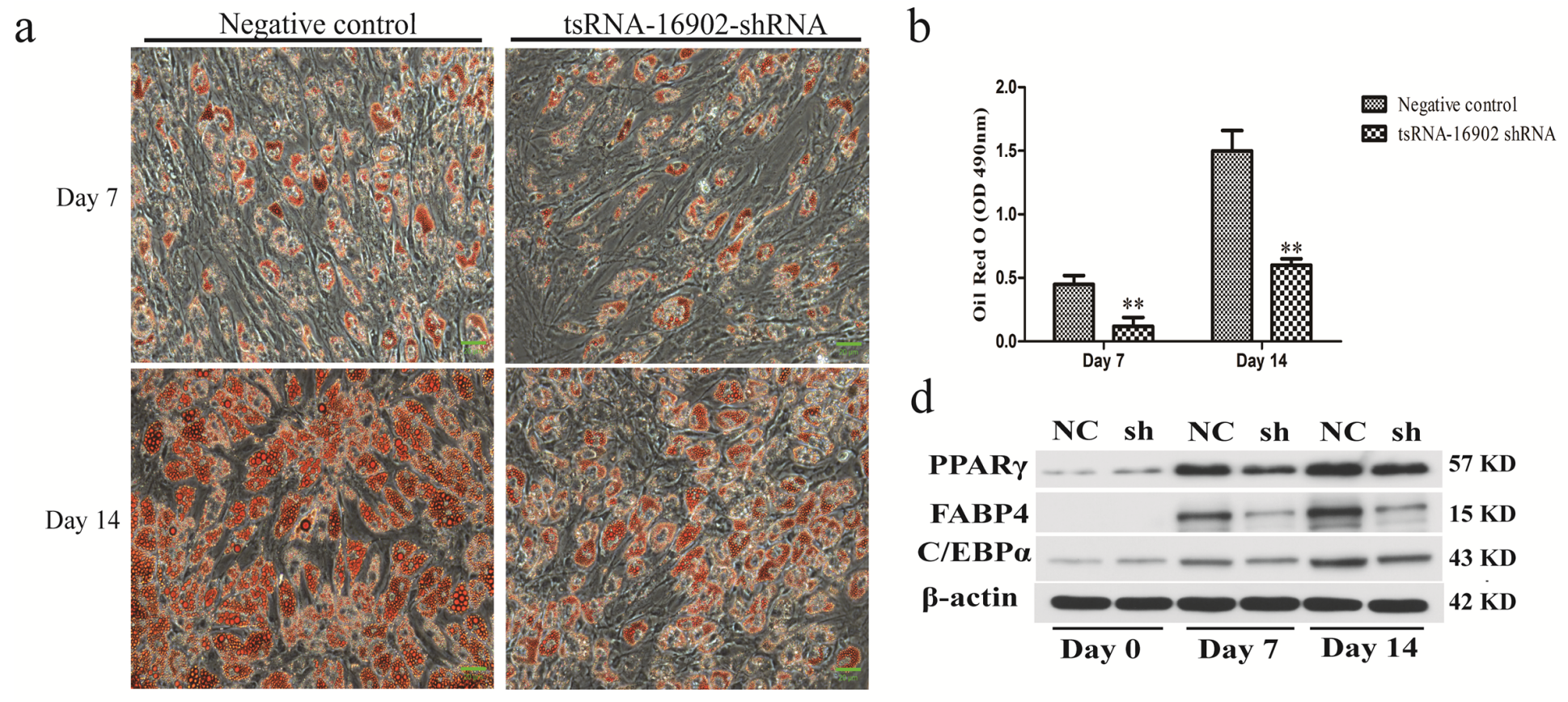

C
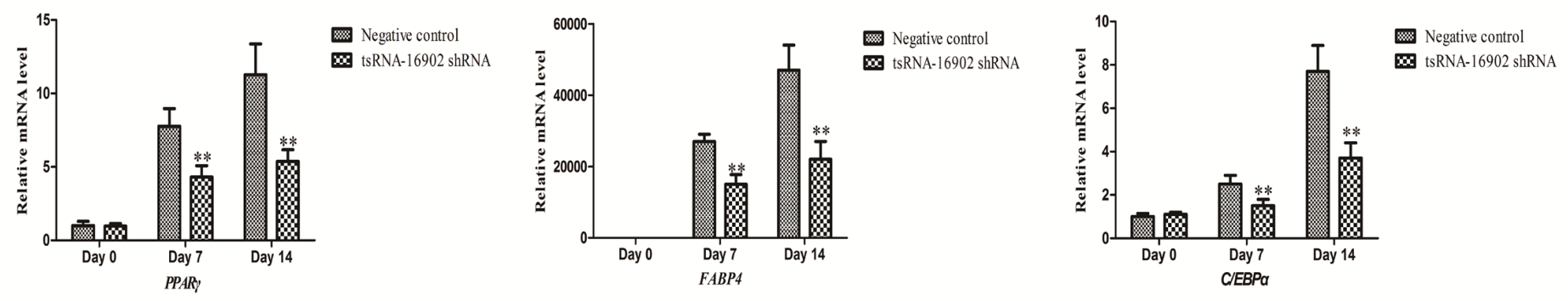

\section{Figure 5}

Knocking down tsRNA-16902 leads to impaired the adipogenic differentiation of these hMSCs after 0, 7, and 14 days. a hMSCs subjected to oil red 0 staining were assessed via light microscope at different time points (20x); scale bar, $20 \mu \mathrm{m}$. b A microplate reader was used in order to quantify oil Red 0 staining intensity. c PPARy, FABP4, C/EBPa, and RARy expression was analyzed using qRT-PCR. d PPARy, FABP4, $C / E B P a$, and RARy expression was analyzed via Western blotting. Data are means $\pm S D(n=3) .{ }^{*} P<0.01$ vs. negative controls, respectively. Note: NC: negative control; sh: tsRNA-16902 shRNA. 
a

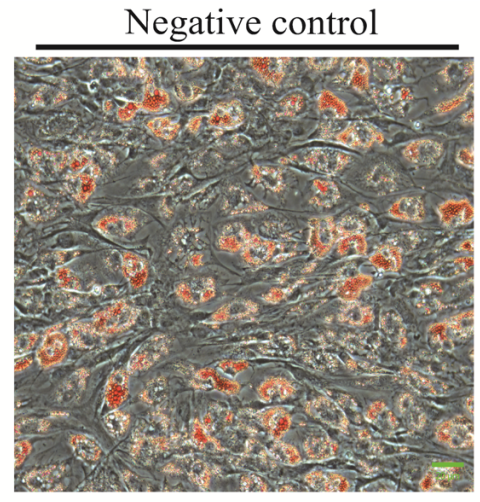

Day 7

Day 14
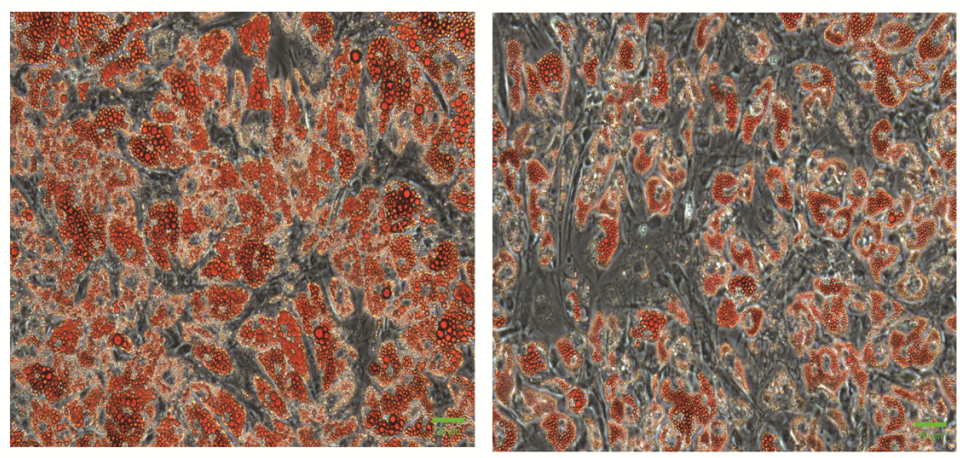

$\mathrm{b}$

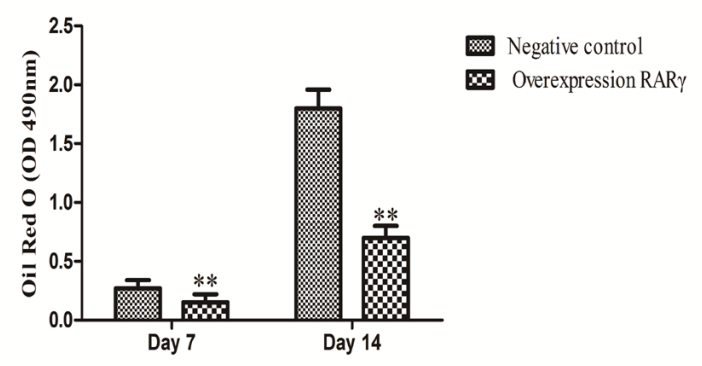

d

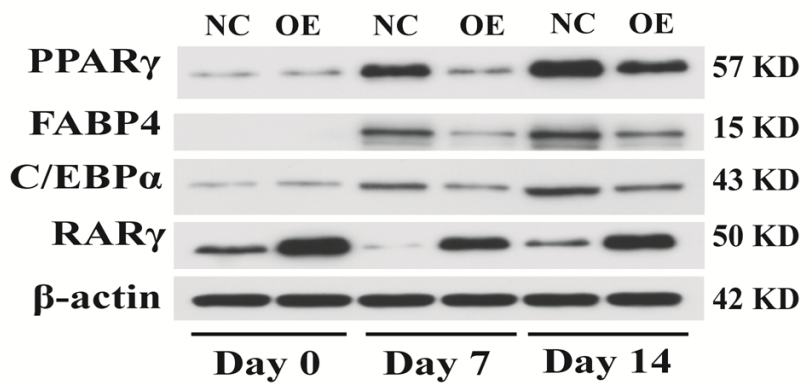

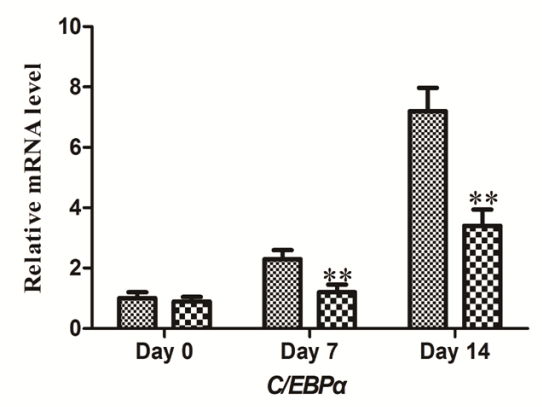

Negative control

$\mathbf{0}$ Overespression RAR $\gamma$

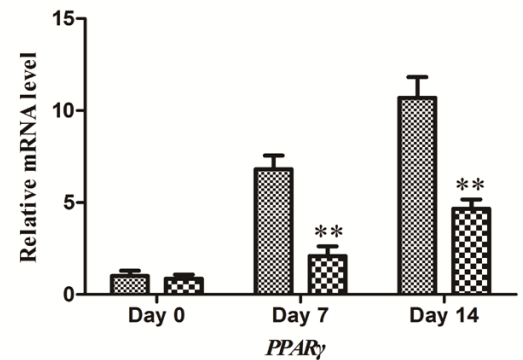

Negative control

$\$$ Overespression RAR $\gamma$

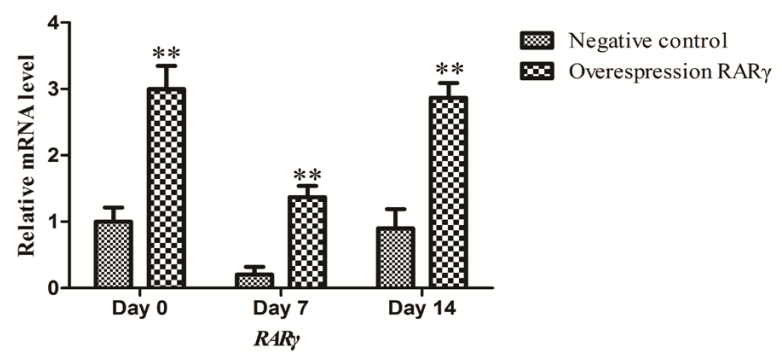

Figure 6

Overexpressing RARy leads to impaired hMSC adipogenesis on days 0,7 and 14. a hMSCs subjected to oil red $O$ staining were assessed via light microscope at different time points (20x); scale bar, 20 $\mu \mathrm{m}$. b A microplate reader was used in order to quantify oil Red 0 staining intensity. c PPARy, FABP4, C/EBPa, and RARyexpression was analyzed using qRT-PCR. d PPARy, FABP4, C/EBPa, and RARy expression was analyzed via Western blotting. Data are means $\pm S D(n=3)$. ${ }^{\star *} P<0.01 v s$. negative controls, respectively. Note: NC: negative control; OE:overexpression RARY. 
$\mathbf{a}$
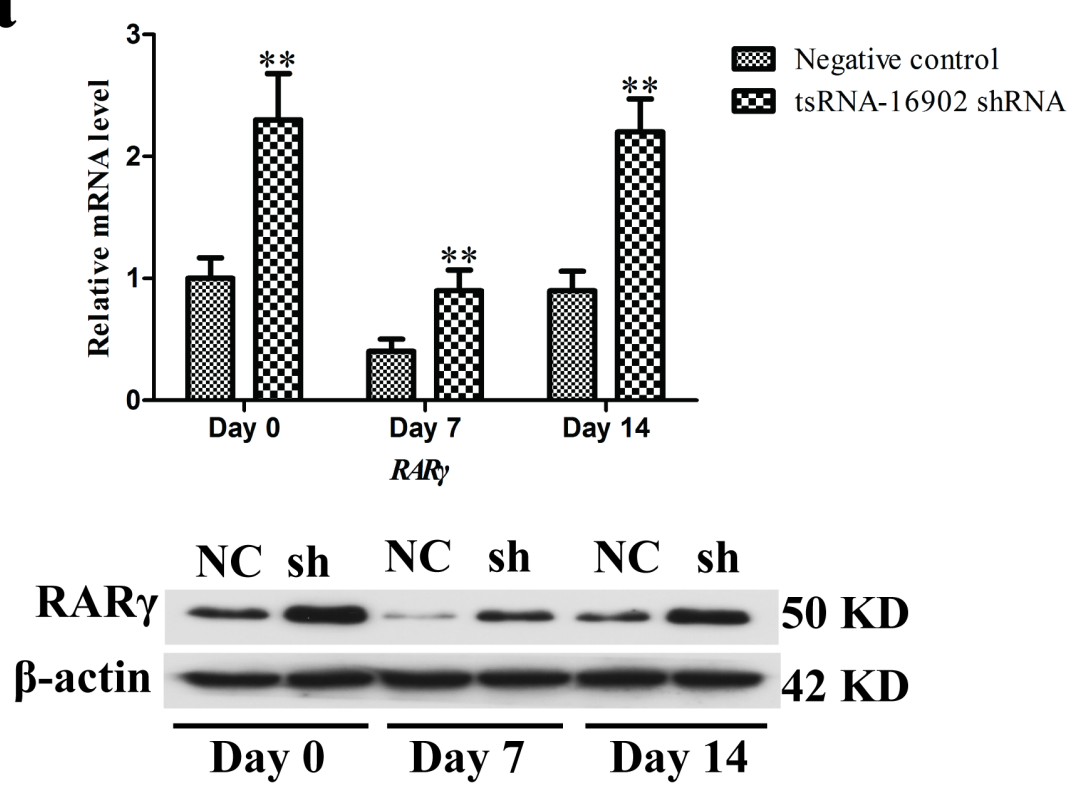

b

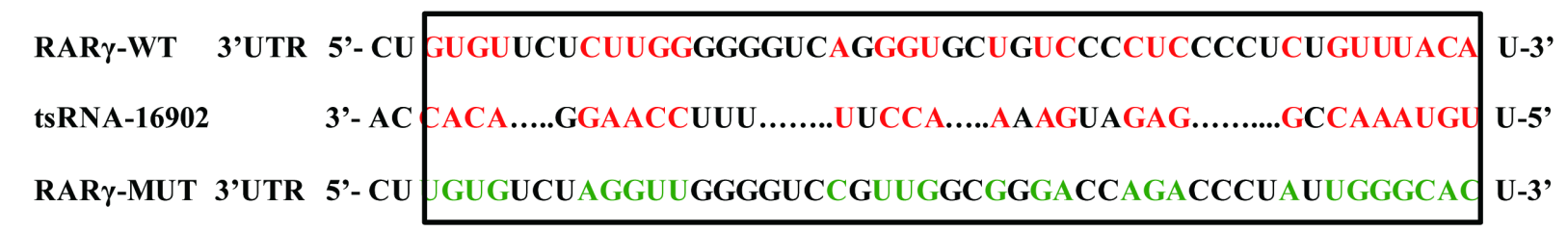

C

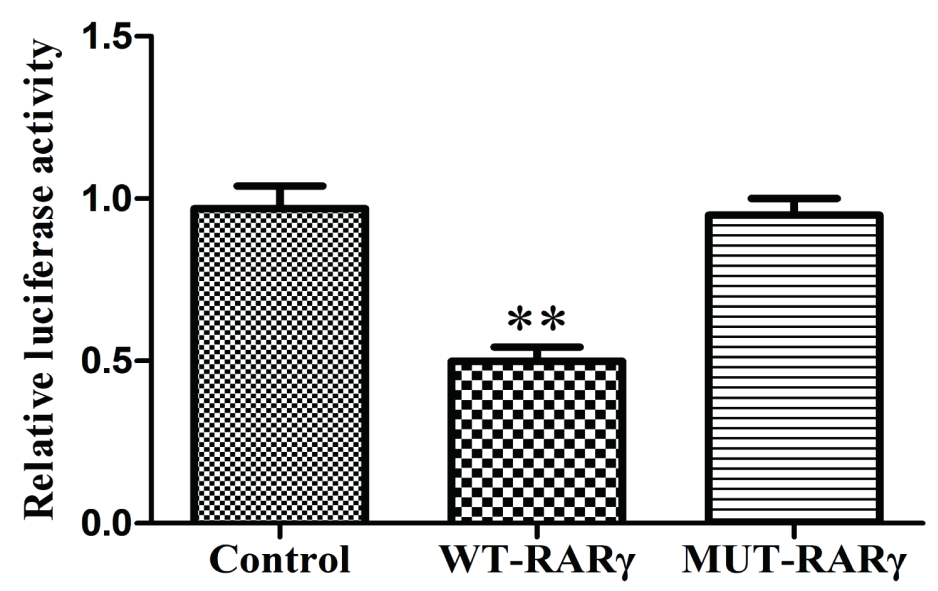

Figure 7

tsRNA-16902 is able to bind to the RARY 3'-UTR. a An analysis of how tsRNA-16902 influenced RARY expression in the context of hMSC adipogenesis on days 0,7 and 14. b An illustration of the putative site of tsRNA-16902 binding within the 3'-UTR of RARy, with green used to highlight those nucleotides mutated for a mutant version of this sequence. c Luciferase activity assay revealed that tsRNA-16902 transfection into cells resulted in a 50\% reduction in luciferase activity for constructs containing a WT but 
not a mutated version of this RARY 3'-UTR binding site. Data are means $\pm S D(n=3)$. **P<0.01 vs. negative controls, respectively. Note: NC: negative control; sh: tsRNA-16902 shRNA.

$\mathbf{a}$
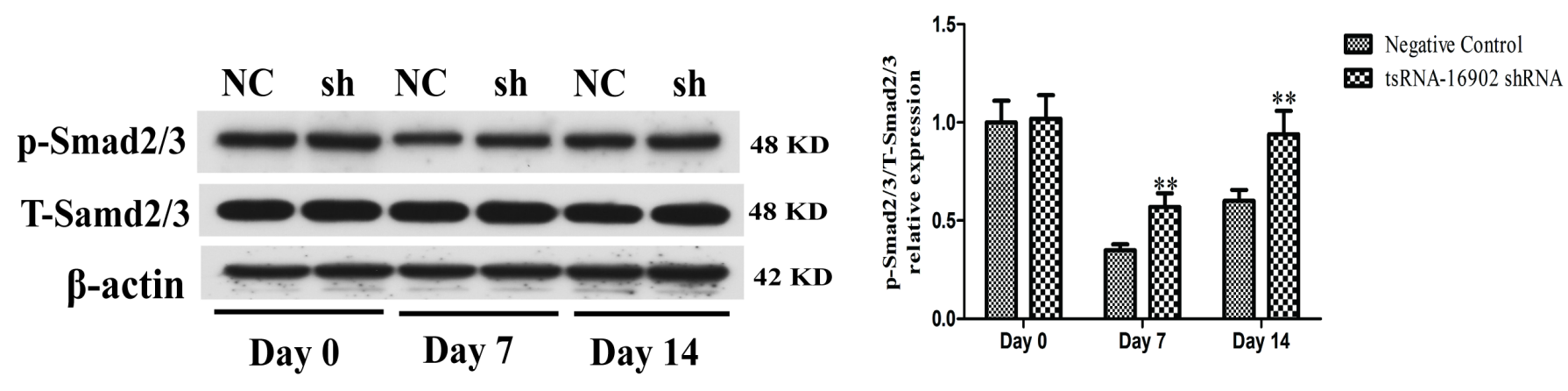

b
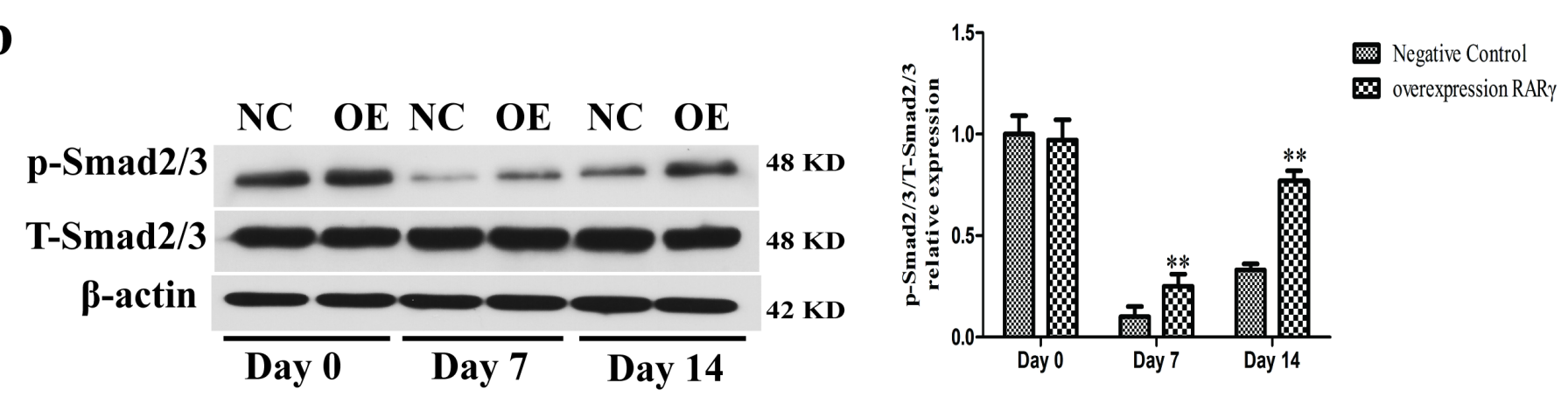

c
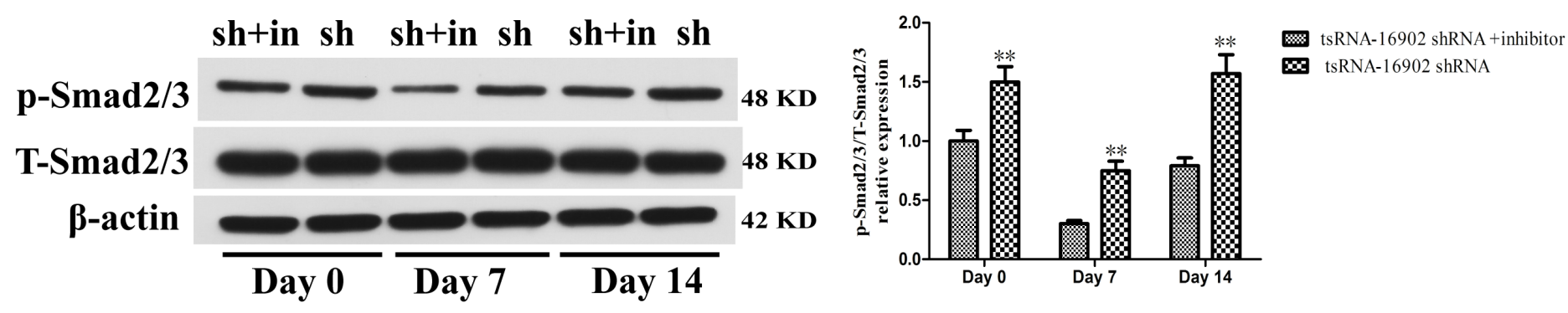

Figure 8

Knockdown of tsRNA-16902 or overexpression of RARy leads to increased p-Smad2/3 levels, and addition of a Smad2/3 inhibitor reduced p-Smad2/3 levels in the context of adipogenesis as determined by measuring Smad2/3 phosphorylation by Western blotting on days 0, 7 and 14. a shRNA-mediated knockdown of tsRNA-16902 alters p-Smad2/3 levels in the context of adipogenic differentiation. b RARY overexpression alters p-Smad2/3 levels in the context of adipogenic differentiation. c Smad2/3 inhibition reduced $p-S m a d 2 / 3$ levels during the adipogenic differentiation of hMSCs. Data are expressed as means $\pm S D(X \pm S D, n=3)$. ${ }^{*} P<0.01 v s$. negative controls, respectively(Fig.8a-b); ${ }^{* * P<0.01 v s . ~ t s R N A-16902 ~}$ shRNA +inhibitor (Fig.8c). Note: sh:shRNA;sh+inh:sh+inhibitor. Note: NC: negative control; sh: tsRNA16902 shRNA; OE:overexpression RARy; sh+in: tsRNA-16902 shRNA +inhibitor; p-Smad2/3: Smad2/3 phosphorylation; T-Smad2/3: Total Smad2/3. 
$\mathrm{a}$

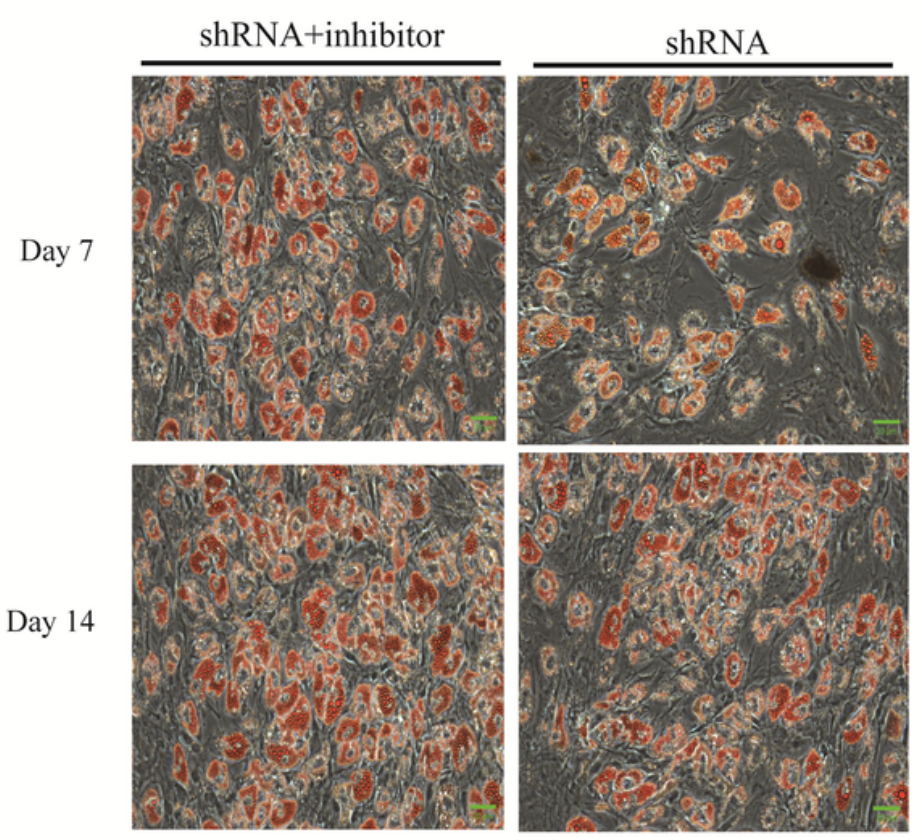

$\mathrm{b}$

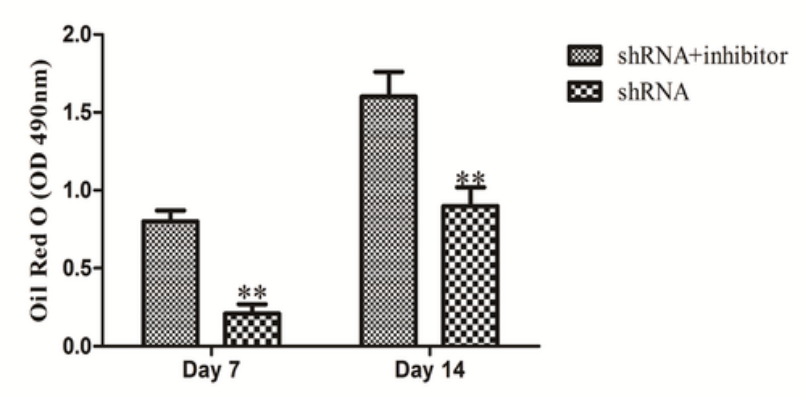

d

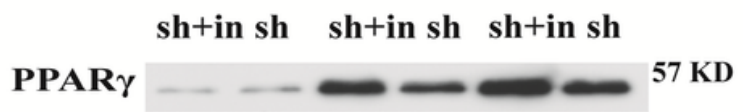

FABP4 $-\longrightarrow-15 \mathrm{KD}$

C/EBPa - - - - $-43 \mathrm{KD}$

$\beta$-actin $\longrightarrow \longrightarrow \longrightarrow$

Day $0 \overline{\text { Day } 7} \overline{\text { Day } 14}$

$\mathrm{c}$
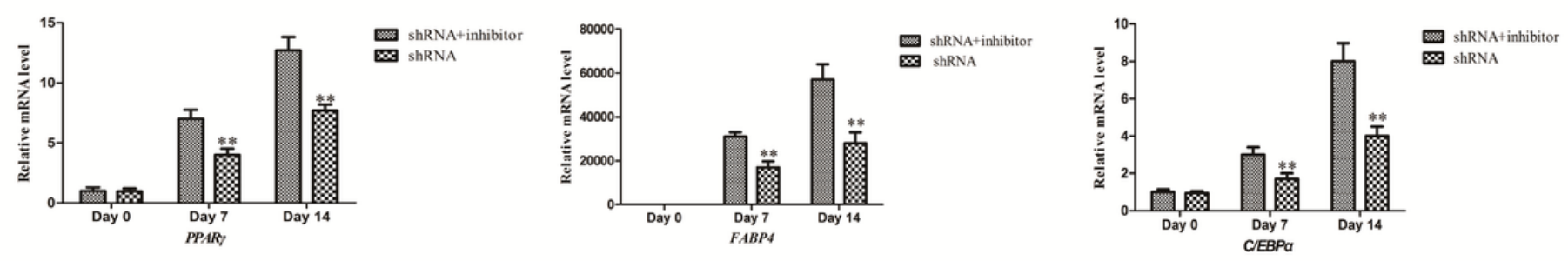

\section{Figure 9}

The adipogenic differentiation of hMSCs in which tsRNA-16902 was knocked down and to which an Smad2/3 signaling inhibitor was added. Oil red 0 staining was employed as a means of assessing lipid

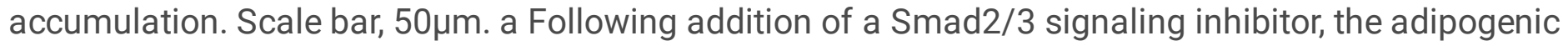
differentiation of hMSCs in which tsRNA-16902 was also knocked down was associated with increased lipid accumulation relative to in cells in which tsRNA-16902 was knocked down but to which no inhibitor was added. $b$ Differences in Oil red $O$ staining intensity were significant between shRNA+inhibitor and shRNA groups. c qRT-PCR was used to compare PPARy, FABP4, and CEBP/a expression in the different groups. d PPARY, FABP4, and C/EBPaprotein levels were assessed via Western blotting. Data are expressed as means $\pm S D(X \pm S D, n=3)$. ${ }^{*} P<0.01 v s$. shRNA+inhibitor, respectively. Note: sh: tsRNA-16902 shRNA; sh+in: tsRNA-16902 shRNA +inhibitor. 


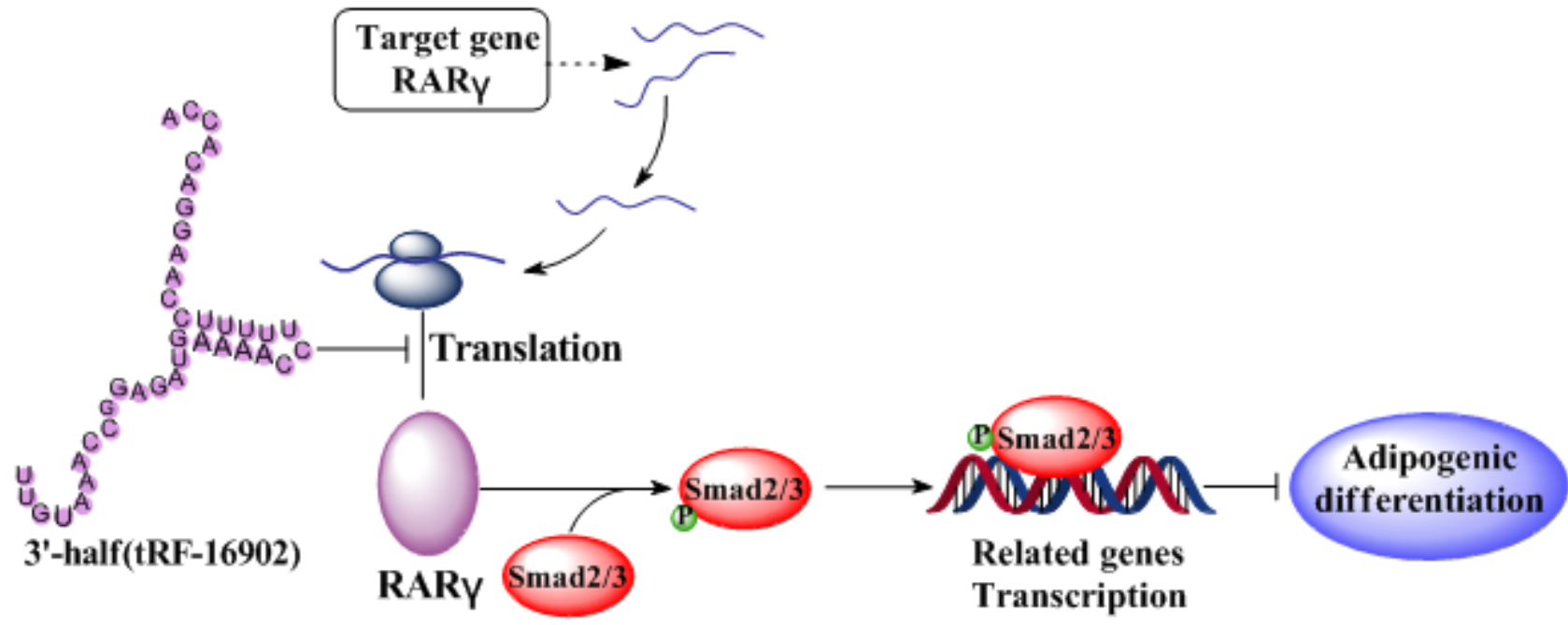

Figure 10

A potential model wherein tsRNA-16902 controls hMSC adipogenesis by targeting of RARY via the Smad2/3 signaling pathway. Note: inhibition; promotion.

\section{Supplementary Files}

This is a list of supplementary files associated with this preprint. Click to download.

- TableS1Primersusedinthisstudy.docx

- FigureS1Sequencingdataanalysisflowchart.pdf

- SupplementaryMaterialFileAlltsRNAdata.pdf

- DentificationofhMSCsviaflowcytometryFileS1.docx

- 6. tif

- 3.tif

- 5.tif

- 7.tif

- 2.tif

- 1.tif

- 8.tif

- 9.tif

- 11.tif

- 4.tif

- 10.tif 
- 12.tif

- 13.tif

- 16.tif

- 14.tif

- 17.tif

- 15.tif

- 20.tif

- 19.tif

- 18.tif

- 21.tif

- 22.tif

- 24.tif

- 23.tif 